\title{
Pain in preterm infants: effects of sex, gestational age, and neonatal illness severity
}

\author{
Beatriz Oliveira Valeri and Maria Beatriz Martins Linhares \\ Universidade de São Paulo, Ribeirão Preto, SP, Brazil
}

\begin{abstract}
Neonates hospitalized in a neonatal intensive care unit are exposed to many painful and stressful procedures. Biobehavioral pain reactivity in preterm infants during the neonatal period may reflect the capacity of the central nervous system to regulate arousal and neurobiological organization. We review empirical studies on the effects of sex, gestational age, and neonatal illness severity on pain reactivity in children born preterm. A literature search was conducted using PubMed, Institute of Scientific Information Web of Science, PsycINFO, Latin American and Caribbean Health Sciences Literature, and Scientific Electronic Library Online databases. Additionally, a special search was performed in online journals that publish pain studies including Pain, Early Human Development, European Journal of Pain, and Pain Management Nursing. The literature search covered the period from 2004 to 2009. Data were extracted according to predefined inclusion and exclusion criteria. Of the 18 studies reviewed, 16 analyzed gestational age, 13 examined neonatal illness severity, and eight focused on sex. Most of the studies analyzed more than one of these three variables. The majority of the studies found effects of gestational age $(n=14)$ and neonatal illness severity $(n=11)$ on pain responses. Only two studies found an influence of sex on infant pain responses. In conclusion, gestational age and neonatal illness severity influence pain responses in infants born preterm. Further studies should be conducted to examine the influence of sex on pain responses. Keywords: pain responses; neonates; sex; gestational age; illness severity.
\end{abstract}

Received 18 November 2011; received in revised form 2 March 2012; accepted 7 March 2012. Available online 29 June 2012.

\section{Introduction}

Studies on the neurobiology of pain have shown that the immaturity of sensory processing in newborns leads to lower thresholds for excitation and sensitization in the spinal cord, thus maximizing the central effects of tissue-damaging inputs (Fitzgerald \& Walker, 2009). Early damage in infancy can lead to prolonged structural and functional alterations in pain pathways throughout the individual's lifespan (Beggs \& Fitzgerald, 2007).

Preterm neonates perceive painful stimuli and exhibit biobehavioral reactivity to pain reflected by facial activity (Stevens, Ridell, Oberlander, \& Gibbins, 2007a), body movements (Holsti, Grunau, Oberlander, \& Whitfield, 2004), physiological responses (Grunau, Haley, Whitfield, Weinberg, Yu, \& Thiessen, 2007), and

Beatriz Oliveira Valeri and Maria Beatriz Martins Linhares, Laboratory of Research in Prevention for Developmental and Behavioral Disorders in Childhood, Department of Neurosciences and Behavior, Faculty of Medicine, Ribeirão Preto, University of São Paulo, Brazil. Correspondence regarding this article should be directed to: Maria Beatriz Martins Linhares, Avenida Tenente Catão Roxo, 2650, Prédio da Saúde Mental (salas 52/53), Faculdade de Medicina, Ribeirão Preto-USP, Campus Universitário Monte AlegreUSP, Ribeirão Preto, SP 14051-140, Brazil. Phone: +55 163602 4610. Fax: +55 16 3602-4504. E-mail: linhares@fmrp.usp.br cortical activity (Bartocci, Bergqvist, Lagercrantz, \& Anand, 2006; Slater et al., 2010).

Maturity of the preterm infant influences the responses to acute pain procedures during hospitalization in the neonatal intensive care unit (NICU; Holsti, Gunau, \& Shany, 2011). In these neurophysiologically immature neonates with low gestational age, discriminating specific sensory changes caused by neonatal pain exposure from the more generalized effects of cumulative pain on multiple aspects of biobehavioral reactivity is challenging (Grunau $\& \mathrm{Tu}, 2007)$. Extremely low-birth-weight infants present inconsistencies in behavioral and physiological pain responses and an inability to sustain responses over time. These inconsistencies reflect the poor regulation of central nervous system development (Stevens et al., 2007a). Gestational age and pain reactivity are highly correlated (Johnston, Fernandes, \& Campbell-Yeo, 2011).

Additionally, preterm infants with a low gestational age present different levels of illness severity. A vulnerable preterm infant's clinical status can be measured using a set of neonatal clinical risk indices including the Clinical Risk Index for Babies (CRIB; International Neonatal Network; Cockburn et al., 1993), CRIB-II (Parry, Tucker, \& Tarnow-Mordi, 2003), Score for Neonatal Acute Physiology (SNAP; Richardson, Gray, McCornick, Workman, \& Goldmann, 1993), Score for Neonatal Acute Physiology-Perinatal Extension II 
(SNAPPE-II; Richardson, Corcoran, Escobar, \& Lee, 2001), Neonatal Medical Index (NNMI; Korner et al., 1993), Neonatal Therapeutic Intervention Scoring System (NTISS; Gray, Richardson, McCormick, Workman-Daniels, \& Goldmann, 1992), and Apgar score during the first 5 min of postnatal age (Apgar, 1953). Clinical risk scores demonstrate better performance than the single birth weight variable in predicting mortality (Zardo \& Procianoy, 2003). CRIB and CRIB-II scores showed greater discriminatory ability than SNAPPE-II in a cohort of infants weighing less than $1,500 \mathrm{~g}$ at birth (Gagliardi et al., 2004). A CRIB index score $\geq 4$ (cut-off) was a better predictor of mortality than birth weight and gestational age (Brito, Matsuo, Gonzalez, Carvalho, \& Ferrari, 2003). As suggested by Gibbins et al. (2008a), neonatal illness severity has been scarcely explored with regard to infant pain responses.

Regarding sex, a previous study found higher pain sensitivity (i.e., lower thresholds, greater discrimination, higher pain ratings, and less tolerance to noxious stimuli) in female than in male adults with differential cortical activation dependent on the type of situational variable (Derbyshire, 2008). However, little conclusive evidence has been provided in regard to sex differences in pain responses in children born extremely preterm, especially at an early age.

To better understand the factors that influence pain reactivity in preterm infants, it is useful to analyze the effects of individual variables such as gestational age, clinical health status, and sex. Concerning the relevance of the effects of individual variables to infant pain reactivity - recovery responses, the present review analyzed empirical studies published between 2004 and 2009 on pain reactivity in children born preterm, with a specific focus on the effects of sex, gestational age, and neonatal illness severity.

\section{Methods}

In 2009 we performed a systematic search of PubMed, PsycINFO, Institute of Scientific Information Web of Science, Latin American and Caribbean Health Sciences Literature, and Scientific Electronic Library Online databases. Moreover, an additional search was performed in some journals that usually publish pain studies including Pain, Early Human Development, European Journal of Pain, and Pain Management Nursing to ensure that as many papers as possible that met the inclusion criteria were included.

According to the objective of this review, the survey was performed with the following keywords: neonates and pain responses and sex; neonates and pain responses and gestational age; neonates and pain responses and severity of illness. The inclusion criteria were the following: empirical studies published in the last 5 years (2004-2009); studies published in English, Spanish, or Portuguese; and studies that included children born preterm. Exclusion criteria were the following: review studies; letters; psychometric studies; studies with samples that did not include preterm infants; and studies that did not analyze the association between pain responses and sex, gestational age, or illness severity.

A total of 95 studies were found that met the inclusion criteria. Of these 95 studies, the following studies were excluded: psychometric studies $(n=9)$; studies in which the results did not analyze sex, gestational age, or illness severity $(n=60)$; and studies in which the assessment did not occur during the painful procedure $(n=8)$. After applying these exclusion criteria, 18 studies remained and were analyzed in the present review.

\section{Results}

\section{Study features}

Of the total sample of 18 studies, eight analyzed sex, 16 analyzed gestational age, and 13 analyzed neonatal illness severity. Concerning the methodological designs of the studies, seven were cross-sectional, four were cross-over, and seven were longitudinal. Table 1 shows the associations between the study design and the study variables.

Table 1 also shows that only three of the 18 studies analyzed the three variables (i.e., sex, gestational age, and illness severity) simultaneously (Holsti et al., 2004; Allegaert, Devlieger, Bulckaert, Naulaers, Casaer, \& Tibboel, 2005; Walker, Franck, Fitzgerald, Myles, Stocks, \& Marlow, 2009). Two studies focused on only one variable (illness severity; Stevens et al., 2007b; Gibbins et al., 2008b). Thirteen studies analyzed two variables simultaneously: five studies analyzed gestational age and sex (Holsti, Grunau, Oberlander, \& Whitfield, 2005; Holsti, Grunau, Whifield, Oberlander, \& Lindh, 2006; Bartocci et al., 2006; Gibbins, Stevens, Beyene, Chan, Bagg, \& Asztalos, 2008c), and eight studies analyzed gestational age and illness severity (Evans, McCartney, Lawhon, \& Galloway, 2005; Grunau et al., 2005; Hermann, Hohmeister, Demirakça, Zohsel, \& Flor, 2006; Stevens et al., 2007c; Rautava et al., 2007; Goffaux, Lafrenaye, Morin, Patural, Demers, \& Marchand, 2008; Williams, Khattak, Garza, \& Lasky, 2009; Hohmeister, Demirakça, Zohsel, Flor, \& Hermann, 2009).

The cross-sectional and cross-over design studies $(n=11)$ focused on clinical pain during the neonatal phase when the infants were hospitalized in the NICU (Holsti et al., 2004, 2005, 2006; Evans et al. 2005; Grunau et al., 2005; Bartocci et al., 2006; Stevens et al., 2007b,c; Gibbins et al., 2008a,c; Williams et al., 2009). One study was a prospective-longitudinal design that examined the association between clinical pain in the NICU and the later pain reactivity of the children at 6 months of age (Gibbins et al., 2008b). Two retrospective-longitudinal studies analyzed the association between history of clinical pain experience in the NICU and pain during later immunization during the first postnatal year (Allegaert et al., 2005; Rautava et al., 2007). Four retrospective-longitudinal studies analyzed the associations between previous neonatal 
Table 1. Study design, number of studies, references, and study variables

\begin{tabular}{|c|c|c|c|c|c|}
\hline \multirow{2}{*}{ Study design } & \multirow{2}{*}{$n$} & \multirow{2}{*}{ Reference } & \multicolumn{3}{|c|}{ Study variables } \\
\hline & & & Sex & Gestational age & Illness severity \\
\hline \multirow[t]{7}{*}{ Cross-sectional } & 7 & 1. Holsti et al. (2004) & $\mathrm{Y}$ & $\mathrm{Y}$ & $\mathrm{Y}$ \\
\hline & & 2. Evans et al. (2005) & $\mathrm{N}$ & $\mathrm{Y}$ & $\mathrm{Y}$ \\
\hline & & 3. Bartocci et al. (2006) & $\mathrm{Y}$ & $\mathrm{Y}$ & $\mathrm{N}$ \\
\hline & & 4. Stevens et al. (2007b) & $\mathrm{N}$ & $\mathrm{N}$ & $\mathrm{Y}$ \\
\hline & & 5. Stevens et al. (2007a) & $\mathrm{N}$ & $\mathrm{Y}$ & $\mathrm{Y}$ \\
\hline & & 6. Gibbins et al. (2008c) & $\mathrm{Y}$ & $\mathrm{Y}$ & $\mathrm{N}$ \\
\hline & & 7. Williams et al. (2009) & $\mathrm{N}$ & $\mathrm{Y}$ & $\mathrm{Y}$ \\
\hline \multirow[t]{4}{*}{ Cross-over } & 4 & 1. Holsti et al. (2005) & $\mathrm{Y}$ & $\mathrm{Y}$ & $\mathrm{N}$ \\
\hline & & 2. Grunau et al. (2005) & $\mathrm{N}$ & $\mathrm{Y}$ & $\mathrm{Y}$ \\
\hline & & 3. Holsti et al. (2006) & $\mathrm{Y}$ & Y & $\mathrm{N}$ \\
\hline & & 4. Gibbins et al. (2008c) & $\mathrm{Y}$ & $\mathrm{Y}$ & $\mathrm{N}$ \\
\hline \multirow[t]{3}{*}{ Longitudinal } & 3 & 1. Allegaert et al. (2005) & $\mathrm{Y}$ & $\mathrm{Y}$ & $\mathrm{Y}$ \\
\hline & & 2. Rautava et al. (2007) & $\mathrm{N}$ & $\mathrm{Y}$ & $\mathrm{Y}$ \\
\hline & & 3. Gibbins et al. (2008b) & $\mathrm{N}$ & $\mathrm{N}$ & $\mathrm{Y}$ \\
\hline \multirow[t]{4}{*}{ Retrospective longitudinal } & 4 & 1. Hermann et al. (2006) & $\mathrm{N}$ & $\mathrm{Y}$ & $\mathrm{Y}$ \\
\hline & & 2. Goffaux et al. (2008) & $\mathrm{N}$ & $\mathrm{Y}$ & $\mathrm{Y}$ \\
\hline & & 3. Walker et al. (2009) & $\mathrm{Y}$ & Y & $\mathrm{Y}$ \\
\hline & & 4. Hohmeister et al. (2009) & $\mathrm{N}$ & $\mathrm{Y}$ & $\mathrm{Y}$ \\
\hline
\end{tabular}

$n$, number of studies; $\mathrm{Y}$, yes; $\mathrm{N}$, no.

pain history and later pain, which was assessed in a laboratory setting in children between 7 and 14 years of age (Hermann et al., 2006; Goffaux et al., 2008; Walker et al., 2009; Hohmeister et al., 2009).

\section{Study findings}

Study results were organized into three blocks according to the main variables: sex, gestational age, and illness severity. Additionally, results were separated in each block according to type of pain including clinical pain, immunization pain, and experimental pain. Clinical pain included studies in which infant pain responses were assessed during the neonatal phase. Immunization pain included studies in which pain was assessed during immunization procedures during the first postnatal year in children born preterm. Experimental pain included studies that associated early pain experience during hospitalization in the NICU and later experimental pain responses in teenagers born preterm.

\section{Sex studies}

\section{Clinical pain}

In six studies that investigated clinical pain responses during the neonatal phase and sex differences, the majority of studies found no significant sex differences (Holsti et al., 2004, 2006; Gibbins et al., 2008a,c). Only two studies found differences between male and female preterm newborns (Bartocci et al., 2006; Holsti et al., 2005), showing more pronounced pain responses in males than in females.

In a cross-sectional study, Bartocci et al.(2006) found sex differences in infants during a painful venipuncture procedure for blood collection. The pain response was greater in male preterm neonates than in female preterm neonates born at 28-36 weeks of gestational age. Males showed a more pronounced increase in bilateral cortical activation compared with females, and this difference was greater in the left hemisphere when venipuncture occurred on the right hand.

In a cross-over study, Holsti et al. (2005) compared two groups of preterm infants with different gestational ages ( $<30$ weeks $v s$. $\geq 30$ weeks; gestational age, 24-32 weeks) during sessions of painful (i.e., heel lance) and stressful (i.e., clustered care) procedures. Two orders for data collection were established: clustered care after rest $v s$. clustered care after pain. Male infants maintained behavioral responses to stress cues assessed by the Newborn Individualized Developmental Care and Assessment Program (NIDCAP) into the recovery phase of the clustered care after pain (i.e., the heel lance procedure).

\section{Immunization pain}

Only one study analyzed sex differences in pain responses in preterm infants during the first postnatal year. Physiological and behavioral pain responses were analyzed during an immunization procedure in children born preterm (gestational age, 25-32 weeks) conducted 34-90 weeks postnatally (Allegaert et al., 2005). No correlations were found between sex and these pain responses.

\section{Experimental pain}

A retrospective-longitudinal study compared teenagers born preterm $(n=43$; gestational age at birth, $<25$ weeks and 6 days) and teenagers born full-term when they were 11 years old. The painful stimulus procedures assessed mechanical and thermal nociception thresholds. Significant differences were found between male and female teenagers. Thermal 
assessment was composed of four types of perception thresholds: cool, warm, cold, and hot. Males in the fullterm control group were more sensitive to cold and hot than females, but the thresholds for cool and warm did not significantly differ between groups. The findings showed that sex in the extremely preterm group did not influence thermal pain thresholds.

\section{Gestational age studies}

Sixteen studies analyzed the effect of gestational age on pain responses. Fourteen studies found significant associations between gestational age and pain responses (Holsti et al., 2004, 2005, 2006; Grunau et al., 2005; Evans et al., 2005; Allegaert et al., 2005; Bartocci et al., 2006; Hermann et al., 2006; Rautava et al., 2007; Gibbins et al., 2008a; Goffaux et al., 2008; Williams et al., 2009; Walker et al., 2009; Hohmeister et al., 2009). Two studies found no significant effects of gestational age on infant pain responses (Stevens et al., 2007c; Gibbins et al., 2008c).

\section{Clinical pain}

In a cross-sectional study of 40 preterm infants (gestational age, 28-36 weeks), Bartocci et al. (2006) found that the increased magnitude of pain-induced cortical activation was negatively correlated with gestational age during a venipuncture procedure for blood collection. Additionally, increased cortical activation after the painful procedure was positively correlated with postnatal age.

Preterm and full-term newborns were studied in four different groups based on gestational age $\left(\mathrm{G}_{1}, 23-\right.$ 27 weeks; $\mathrm{G}_{2}, 28-31$ weeks; $\mathrm{G}_{3}, 32-35$ weeks; $\mathrm{G}_{4}, 36-$ 38 weeks; Gibbins et al., 2008a). Less mature infants ( $G_{1}$ group) showed fewer changes in total facial activity responses from baseline in response to a heel lance procedure for blood collection compared with the $\mathrm{G}_{3}$ and $\mathrm{G}_{4}$ groups. Infants in the $\mathrm{G}_{1}$ group had lower minimum, mean, and maximum oxygen saturation levels compared with infants in the other groups during three phases of blood collection (i.e., baseline, lance, and return-tobaseline phases). When the authors compared mean oxygen saturation differences between baseline and lance phases, infants born at 28-31 weeks of gestational age $\left(\mathrm{G}_{2}\right.$ group) exhibited the greatest changes, and infants born at $32-35$ weeks of gestational age ( $\mathrm{G}_{3}$ group $)$ exhibited the fewest changes. Concerning heart rate, $\mathrm{G}_{1}$ and $\mathrm{G}_{2}$ groups had significantly higher minimum, mean, and maximum heart hates during baseline compared with the two more mature groups $\left(\mathrm{G}_{3}\right.$ and $\mathrm{G}_{4}$ groups). This heart rate difference was maintained during the lance and return-to-baseline (recovery) phases with the $\mathrm{G}_{1}$ and $\mathrm{G}_{2}$ groups presenting significantly higher heart rates compared with the $\mathrm{G}_{4}$ group.

According to the results of Holsti et al. (2004), infants born at a gestational age of $<30$ weeks (gestational age 25-30 weeks of gestational age) showed more behavioral responses (i.e., facial and body activity from the NIDCAP) during a heel lance procedure compared with older infants (gestational age 30-32 weeks of gestational age). Evans et al. (2005) found that, according to a multidimensional pain measure reflected by Premature Infant Pain Profile (PIPP) scores, infants born at 27-30 weeks and infants born at 33-36 weeks of gestational age showed significantly less physiological and behavioral responses during hospitalization in the NICU than infants born at 31-33 weeks of gestational age. The authors attributed the significantly higher PIPP scores in the transitional group to neurological changes that occur at approximately 31 weeks of gestational age.

In a set of cross-over studies, results showed relationships between stressful and painful procedures. A study by Holsti et al. (2005) found that infants born at an earlier gestational age ( $<30$ weeks) were less able to flex their legs and bring their hands to their mouth compared with infants born at later gestational ages ( $\geq 30$ weeks) during the handling phase (i.e., stress) of clustered care following a pain procedure. Moreover, during the recovery phase of both sessions (clustered care following rest and clustered care following pain), infants born at later gestational ages ( $\geq 30$ weeks) continued to exhibit stability in their responses to NIDCAP cues and stress cues during the recovery phase following the clustered care and following rest procedure. The same authors published another study in 2006 and showed that infants born at earlier gestational ages $(<30$ weeks) presented higher facial responses and higher mean heart rates during the recovery phase when pain occurred after clustered care.

A study of the association between painful (i.e., heel lance) and nonpainful (i.e., diaper change) procedures found no differences in total facial activity between infants born at 23-25 weeks (incomplete, with 6/7 days) and 26-27 weeks (incomplete, with 6/7 days) of gestational age (Gibbins et al., 2008c). Each group had more total facial expressions during the procedural phase in both the diaper change and heel lance procedures, but the differences between procedures were not significant. Similarly, no differences in total body movements were found in infants born at 25 (incomplete, with $6 / 7$ days) weeks of gestational age compared with the more mature gestational age group. When examining individual facial activity, greater activity was present in the infants born at 26-27 weeks (incomplete, with 6/7 days) of gestational age, but the differences were not significant. Moreover, no significant differences were found in individual body movements between the gestational age groups.

In conclusion, gestational age studies demonstrated that biobehavioral pain response patterns vary depending on the stratification of gestational age at birth in preterm infant samples. Less mature infants with a low gestational age had fewer behavioral responses and higher physiological responses. Additionally, lower gestational age was associated with higher cortical activation during acute pain experiences. 


\section{Immunization pain}

Infants born preterm have higher minimum fundamental frequencies when they cry during painful immunization procedures in the first postnatal year than infants born full-term (Rautava et al., 2007).

\section{Experimental pain}

A retrospective-longitudinal study examined the efficacy of endogenous descending inhibitory pathways in children born prematurely by associating experimental pain responses with pain experienced during hospitalization in the NICU in children born preterm and in children born full-term at 7- to 11-years of age (Goffaux et al., 2008). Children who suffered more pain during hospitalization (gestational age 24-31 weeks) had higher pain intensity scores than preterm children who had a lower history of pain in the NICU (gestational age 28-32 weeks) and children born full-term (gestational age 38-40 weeks). Cold water immersion procedure was performed to assess the perceived pain and determine differences in pain threshold values in comparison to pain sensitivity. The immersion procedure successfully reduced pain intensity values in the full-term and low-pain preterm groups but had no effect in the high-pain preterm group. The groups did not differ in initial pain sensation, and the region of thermal stimulation (i.e., calf $v s$. forearm) had no effect. Furthermore, before immersing the hand in cold water during the resting phase, the high-pain preterm group had the highest resting heart rate followed by the low-pain preterm group and full-term group. The study assessed the percentage change in heart rate during the immersion procedure, finding qualitatively different responses among groups. The high-pain preterm group showed essentially no change in response to the immersion procedure, whereas the low-pain preterm and full-term groups showed significant increases in heart rates. In the low-pain preterm and full-term groups, this increase remained significant throughout the first $90 \mathrm{~s}$. By the end of the immersion session, heart rate returned to baseline in all three groups.

Walker et al. (2009) compared pain responses at 11 years of age in children born preterm (gestational age $<25$ weeks) and children born full-term. The painful stimulus was applied during both thermal and mechanical tests. Thermal stimulation was applied with a handheld contact thermode to provide uniform contact on the rounded thenar eminence. The children were asked to press a button when they felt a temperature change (cool, warm, cold, or hot). Paradoxical responses were classified as the following: (a) cold felt as hot, (b) hot felt as cold, (c) rapid transition to hot, (d) rapid transition to cold, and (e) other. Mechanical detection threshold was assessed using a filament that touched the skin. Children born extremely preterm were significantly less sensitive than children born full-term to all of the thermal modalities, and this difference was greater for more intense cold and hot stimulation. More children born extremely preterm showed paradoxical or unusual thermal responses compared with the full-term control children.

Children born at $<31$ weeks of gestational age presented greater perceptual sensitization to tonic heat at 9- to 11-years of age than children born at $>37$ weeks of gestational age during thermal perceptual sensitization/heat pain threshold and mechanical perceptual sensitization/mechanical pain threshold procedures (Hermann et al., 2006). These findings indicate habituation to the painful stimulus. At the thenar, children born full-term who remained in the NICU had higher heat pain thresholds than control full-term children. At the trigeminal site, children born preterm had higher heat pain thresholds than the group of children born full-term. No difference was found between groups with regard to mechanical stimulation.

Results of the studies of experimental pain showed that gestational age at birth alters pain thresholds and sensitivity to pain during the teenage period. A lower gestational age was associated with lower pain thresholds and higher pain sensitivity.

\section{Illness severity studies}

Of the total 18 studies reviewed, 13 assessed illness severity (Holsti et al., 2004; Evans et al., 2005; Stevens et al., 2007b,c; Williams et al., 2009; Grunau et al., 2005; Allegaert et al., 2005; Rautava et al., 2007; Gibbins et al., 2008b; Hermann et al., 2006; Goffaux et al., 2008; Walker et al., 2009; Hohmeister et al., 2009). Only three studies found no significant differences in pain responses with regard to the illness severity variable (Stevens et al., 2007c; Hermann et al., 2006; Goffaux et al., 2008).

Illness severity was assessed using several operational definitions. Nine studies used scores of clinical risk for mortality and morbidity to classify illness severity including the CRIB (Walker et al., 2009), SNAP-II (Holsti et al., 2004; Grunau et al., 2005), SNAP-Perinatal Extension (SNAP-PE; Stevens et al., 2007b; Gibbins et al., 2008b), SNAPPE-II (Hermann et al., 2006; Hohmeister et al., 2009), NTISS (Gibbins et al., 2008b; Evans et al., 2005), Neurobiologic Risk Score (NBRS) at discharge (Gibbins et al., 2008b), and Apgar score at the 5th minute (Rautava et al., 2007; Stevens et al., 2007b).

Eleven studies used data of illness severity based on clinical events including the following: high opioid exposure (Holsti et al., 2004; Allegaert et al., 2005), early sickness during the neonatal period (Holsti et al., 2004), number of invasive and painful procedures since birth (Holsti et al., 2004; Hermann et al., 2006; Goffaux et al., 2008), number of repeated heelstick procedures (Evans et al., 2005), length of stay in the NICU or hospitalization (Allegaert et al., 2005; Hermann et al., 2006; Goffaux et al., 2008; Hohmeister et al., 2009; Williams et al., 2009), neonatal surgery and major procedures during NICU stay (Allegaert et al., 2005; Walker et al., 2009), number of days under mechanical ventilation (Allegaert et al., 2005; Rautava et al., 2007; Goffaux et al., 2008; Williams et al., 2009), 
brain abnormities and bronchopulmonary dysplasia (Rautava et al., 2007), and risk for neurological impairment (Stevens et al., 2007b,c; Gibbins et al., 2008b). Risk for neurological impairment was classified as high (perinatal asphyxia, intraventricular hemorrhage [IVH] grade III or IV or a syndrome or chromosomal anomaly), moderate (acute disease processes such as persistent pulmonary hypertension, severe meconium aspiration, meningitis, hydrocephalus, and necrotizing enterocolitis), and low (respiratory distress requiring ventilation and sepsis).

\section{Clinical pain}

Stevens, Gibbins, McGrath, and Franck published two cross-sectional studies in 2007 that compared three different groups of infants born between 25 and 40 weeks of gestational age according to high, moderate, and low risk for neurological impairment. The risk for neurological impairment was based on several factors including congenital syndrome/chromosomal abnormalities (e.g., congenital trisomies), birth trauma (e.g., fractures, nerve injuries), extremely preterm birth (e.g., IVH, necrotizing enterocolitis [NEC]), and acquired illnesses with central nervous system involvement (Robertson, Svenson, \& Joffres, 1998). The groups of neonates were stratified according to neurological impairment score (Stevens et al., 2007b). The group with the highest risk for neurological impairment underwent significantly more painful procedures ( $>15 /$ day) and were administered less opioids than infants with minimal or no risk for neurological impairment.

In the first study, Stevens et al. (2007b) found that the low-risk group exhibited the greatest total facial reactions in the post-procedure phase of the heel lance procedure for blood collection than the moderate- and high-risk groups. Moreover, the moderate-risk group presented higher minimum and mean fundamental frequencies of crying during the heel lance procedure compared with the high-risk group. In the second study, all three groups (high, moderate, and low) exhibited the same underlying structure of pain responses reflected by facial reactions, accounting for the greatest variance across all factors including both behavioral (facial reactions) and physiological (oxygen saturation and heart rate) indicators (Stevens et al., 2007c).

Preterm infants who were exposed to a greater number of invasive procedures since birth and infants who had greater opioid exposure showed more behavioral responses to pain (Holsti et al., 2004). Additionally, infants who were sicker earlier during the neonatal period (SNAP-II on day 1) displayed more facial reactions (Holsti et al., 2004). Grunau et al. (2005) found that greater illness severity (SNAP-II) was significantly associated with lower heart rate variability in the very low gestational age group of infants born between 29 and 32 weeks of gestational age.

Additionally, Williams et al. (2009) found that newborns with higher illness severity assessed by low birth weight, young postmenstrual age at birth (mean 26 weeks of age), mechanical ventilation, and long length of stay in the NICU exhibited less-pronounced behavioral pain responses on the Neonatal Infant Pain Scale (NIPS).

\section{Immunization pain}

A longer length of stay in the NICU was associated with a longer duration of crying during an immunization procedure in infants born preterm, in the first year of age (Allegaert et al., 2005). An Apgar score of $<5$ at the 5 th minute in very-low-birth-weight preterm infants was associated with shorter cry utterances and a longer duration of crying during an immunization procedure at 1.5 years of age (Rautava et al., 2007).

Newborns born at 25-40 weeks of gestational age were compared according to their risk for neurological impairment in two sessions of a painful procedure (Gibbins et al., 2008b). In the first session, infants were assessed at 1-7 days of postnatal age during a heel lance procedure in the NICU. The second session was conducted later during the immunization procedure when the infants were at 6 months of corrected age for prematurity. In both sessions, infants at low risk for neurological impairment exhibited higher facial reaction scores than the moderate- and high-risk groups. Moreover, high-risk infants presented more changes in facial reactions from baseline in the heel lance phase than the other two groups. Infants at moderate risk for neurological impairment had higher mean fundamental crying frequencies in session 1 compared with high-risk infants. This group, in turn, had longer crying bouts in session 2 than moderate-risk infants.

\section{Experimental pain}

Of the four longitudinal pain studies, two assessed children who were born with low gestational age at 9to 14-years of age during painful stimulus procedures (Walker et al., 2009; Hohmeister et al., 2009). Correlations were assessed to determine whether NICU stay influences later pain responses. Changes in the thermal perception of cold and hot were the most extreme in children born extremely premature and who underwent surgery during their NICU stay (Walker et al., 2009). Children born preterm who underwent surgery during childhood had greater externalizing coping strategies compared with children born preterm with no surgical history. Similarly, Hohmeister et al. (2009) found that pain catastrophizing was significantly correlated with illness severity/mortality risk (SNAPPEII) and the duration of hospitalization in children with NICU experience born preterm at 9- to 14-years of age.

\section{Discussion}

The present review provided evidence that individual variables such as gestational age and illness severity influence pain reactivity in vulnerable children born preterm. The neurobehavioral maturity factor reflected by gestational age should be carefully examined with 
regard to its impact on pain assessments. Infant samples in pain studies may be stratified based on gestational age at birth because this variable may cause substantial differences in pain responses. An infant expresses pain perception and the capacity of self-regulation based on the maturity level of the neurological system.

With regard to gestational age, 30 weeks of gestational age may be a relevant cut-off value when assessing differences in infant pain reactivity. Two studies found greater pain responses in the less mature infant groups $(<30$ weeks) than in the more mature groups ( $>30$ weeks; Holsti et al., 2004, 2006). Two other studies with the same gestational age cut-off found decreased behavioral and physiological pain responses in preterm children born at later gestational ages after experiencing painful procedures, suggesting that infants born at $>30$ weeks of gestational age continued to show self-regulatory behaviors (Holsti et al., 2005; Gibbins et al., 2008a). Neurological changes and developmental physiological changes transition from sensitization to habituation with repeated pain at approximately 30 weeks of gestational age in human infants (Fitzgerald, 1995), possibly explain the inconsistent responses concerning this cut-off in gestational age. These results suggest that the responses of preterm infants after painful stimulation should be assessed to better understand the damped responses associated with habituation after cumulative painful stimulus. Furthermore, results showed that pain response assessment in low-gestational-age infants should be based on a multidimensional approach to better detect pain expression. Combined behavioral and physiological dimensions may bolster the evidence of pain reactivity in these infants. Ranger et al. (2007) suggested that age significantly affected the robustness of pain-related behavior including postnatal age at the time of assessment and gestational age at birth.

The longitudinal studies of Goffaux et al. (2008) and Hermann et al. (2006) demonstrated that gestational age is an important factor in physiological pain responses during pain experiences later in childhood. Children who were born more prematurely showed higher scores of thermal perceptual sensitization during pain threshold assessments than children born full-term. Preterm children at 11 years of age were more sensitive to the identification of thermal changes than full-term children (Walker et al., 2009). These results highlight the importance of not only comparing children born preterm and full-term but also analyzing gestational age stratified over medium-term consequences.

Illness severity also requires further investigation. The results showed inconsistencies with regard to the multiple definitions of infant illness severity assessment. Illness severity based on predetermined mortality/ morbidity risk scores showed inconsistent results between this factor and infant pain responses (Holsti et al., 2004; Grunau et al., 2005; Evans et al., 2005). Holsti et al. (2004) showed that preterm infants with higher SNAP scores displayed more facial twitches, handson-face, and fistings in response to the NIDCAP than preterm infants who were physiologically more stable (Holsti et al., 2004). Moreover, Holsti et al. (2005) found that infants born with a very low birth weight and higher SNAP scores had significantly lower heart rate variability. Divergent results were found when NTISS scores were correlated with PIPP scores (Evans et al., 2005). A more clinically severe status (measured by NTISS) was associated with lower PIPP scores. These findings demonstrate the necessity of considering the influence of illness severity. The NIDCAP measures pain responses and stress behaviors. Pain responses were also measured by the PIPP, which is a multidimensional instrument that includes behavioral, physiological, and contextual components of pain assessment.

Holsti et al. (2011) highlighted that, although using a pain scale that combines behavioral with physiological indices into a single score may be simple, clinicians and researchers should consider each domain of an index separately to ensure that the individual components of the pain response are adequately managed. When univariate behavioral scales are used, physiological recordings add important complementary information about specific pain responses.

Only one study correlated Apgar scores at the 5th minute with infant crying (Rautava et al., 2007). Infants with low Apgar scores reflecting more severe clinical status exhibited a shorter duration of crying during a painful procedure.

Assessment of illness severity included an examination of the risk for neurological impairment. Of the three studies that included risk for neurological impairment, two showed positive results (Stevens et al., 2007b; Gibbins et al., 2008b). When infants presented a higher risk for neurological impairment, higher NFCS scores were found during painful procedures (Stevens et al., 2007b). The same study found that infants with a low risk for neurological impairment had higher NFCS scores during a painful procedure than infants with moderate and high risks for neurological impairment. The contrasting results based on risk for neurological impairment may have occurred because of the stratification of the risk levels attributed on the investigation that compared the infants' pain responses according to the three different levels for NI risk.

Another group of studies found significant results based on infant clinical events that occurred during hospitalization (Williams et al., 2009; Allegaert et al., 2005; Walker et al., 2009). Several correlations demonstrated that sicker infants needed more medical interventions during their NICU stay including painful procedures, mechanical ventilation, opioid exposure, surgery, and longer hospitalization. Mechanical ventilation and length of stay were both negatively associated with NIPS pain scores (Williams et al., 2009). Infants born preterm who stayed more days in the NICU exhibited a longer duration of crying during an immunization session at approximately 1 year of postnatal age (Allegaert et al., 2005). Children born preterm who underwent surgery during hospitalization 
in the NICU presented more changes in thermal pain stimulus perception and more externalizing coping strategies at 11 years of age than preterm children who did not require surgery (Walker et al., 2009).

Neonatal illness severity requires further investigation to obtain a precise operational definition to better examine the effect of this variable on infant's pain responses. The studies that assessed neonatal illness severity found associations with pain responses in children born preterm, but several tools were used to assess this variable. The methodological differences between these studies are a limitation that prevents reaching consistent conclusions regarding the effect of neonatal illness severity on pain responses. In addition, the correlation between gestational age, illness severity and cumulative pain exposure must be examined in the analysis of infants' pain reactivity.

The sex factor also requires further investigation with regard to its effects on infant pain responses. Few studies found differences between male and female preterm infants in pain responses during a blood collection procedure (Bartocci et al., 2006; Holsti et al., 2005). The majority of studies showed that both male and female infants have similar patterns of pain responses, suggesting that sex has a unidirectional effect on pain responses (Holsti et al., 2004, 2006; Gibbins, et al., 2008a,c). Moreover, the study that found these differences assessed physiological responses based on brain images (Bartocci et al., 2006). Sex differences were found in responses to stress procedures where male newborns exhibited more stress responses to a manipulation procedure than females (Holsti et al., 2005). The findings of both studies showed sex differences in pain responses reflected by cortical activation as specific pain response and stress reactivity, respectively. Further investigations should assess the effects of sex on pain responses by considering both neurological activation and biobehavioral patterns.

In conclusion, gestational age and neonatal illness severity influence pain responses in infants during the neonatal phase and in children born preterm. The 5-year period for review of the literature may be a narrow window of time and thus excluded relevant studies published prior to this period. Further investigations should better examine the influence of sex on pain responses. Additionally, studies that assess the effect of illness severity on pain responses should be better developed and based on clinical neonatal illness severity scales to allow comparisons among studies. Future studies should address the relationships between gestational age, illness severity and cumulative pain exposure in pain reactivity and recovery in preterm infants.

\section{Acknowledgements}

The authors are grateful for the research support of the State of São Paulo Research Foundation (FAPESP) to $\mathrm{BOV}$ and for a grant from the National Council of Science and Technology Development (CNPq) to MBML.

\section{References}

*Allegaert, K., Devlieger, H., Bulckaert, D., Naulaers, G., Casaer, P., \& Tibboel, D. (2005). Variability in pain expression characteristics in former preterm infants. Journal of Perinatal Medicine, 33, $442-448$.

Apgar, V. (1953). A proposal for a new method of evaluation of the newborn infant. Current Researches in Anesthesia and Analgesia, $32,260-267$.

*Bartocci, M., Bergqvist, L. L., Lagercrantz, H., \& Anand, K. J. S. (2006). Pain activates cortical areas in the preterm newborn brain. Pain, 122, 109-117.

Beggs, S., \& Fitzgerald, M. (2007). Development of peripheral and spinal nociceptive systems. In K. J. S. Anand, B. J. Stevens, \& P. J. McGrath (Eds.), Pain in neonates and infants, 3rd edition (series title: Pain research and clinical management) (pp. 11-24). Philadelphia: Elsevier.

Brito, A., Matsuo, T., Gonzalez, M. R., Carvalho, A. B. R., \& Ferrari, L. S. L. (2003). CRIB score, birth weight and gestational age in neonatal mortality risk evaluation. Revista de SáudePública, 37, $597-602$.

Cockburn, F., Cooke, R. W. I., Gamsu, H. R., Greenough, A., Hopkins, A., Mcintosh, N., Ogstont, S. A., Parryt, G. J., Silverman, M., Shaw, J. C. L., Tarnow-Mordit, W. O., \& Wilkinson, A. R. (1993). The CRIB (Clinical Risk Index for Babies) score: a tool for assessing initial neonatal risk and comparing performance of neonatal intensive care units. Lancet, 342, 193-198.

Derbyshire, S. W. G. (2008). Gender, pain and the brain. Pain: Clinical Updates, XVI(3).

*Evans, J. C., McCartney, E.M., Lawhon, G., \& Galloway, J. (2005). Longitudinal comparison of preterm pain responses to repeated heelsticks. Pediatric Nursing, 31, 216-221.

Fitzgerald, M., \& Walker, S. M. (2009).Infant pain management: a developmental neurobiological approach. Nature Clinical Practice Neurology, 5, 35-50.

Fitzgerald, M. (1995). Pain in infancy: some unanswered questions. Pain Reviews, 2, 77-91

Gagliardi, L., Cavazza, A., Brunelli, A., Battaglioli, M., Merazzi, D., Tandoi, F., Cella, D., Perotti, G. F., Pelti, M., Stucchi, I., Frisone, F., Avanzini, A., \& Bellù, R. (2004). Assessing mortality risk in very low birthweight infants: a comparision of CRIB, CRIB-II, and SNAPPE-II. Archives of Disease in Childhood, 89, F419F422.

*Gibbins, S., Stevens, B., McGrath, P. J., Yamada, J., Beyene, J., Breau, L., Camfield, C., Finley, A., Franck, L., Johnston, C., Howlett, A., McKeever, P., O’Brien, K., \& Ohlsson, A. (2008a). Comparison of pain responses in infants of different gestational ages. Neonatology, 93, 10-18.

*Gibbins, S., Stevens, B., McGrath, P., Dupuis, A., Yamada, J., Beyene, J., ... Ohlsson, A. (2008b). Changes in physiological and behavioural pain indicators over time in preterm and term infants at risk for neurologic impairment.Early Human Development, 84, 731-738.

*Gibbins, S., Stevens, B., Beyene, J., Chan, P. C., Bagg, M., \& Asztalos, E. (2008c). Pain behaviours in Extremely Low Gestational Age infants. Early Human Development, 84, 451-458.

*Goffaux, P., Lafrenaye, S., Morin, M., Patural, H., Demers, G., \& Marchand, S. (2008). Preterm births: can neonatal pain alter the development of endogenous gating systems? European Journal of Pain, 12, 945-951.

Gray, J. E., Richardson, D. K., McCormick, M. C., Workman-Daniels, K., \& Goldmann, D. A. (1992). Neonatal therapeutic intervention scoring system: a therapy-based severity-of-illness index. Pediatrics, 90, 561-567.

*Grunau, R. E., Holsti, L., Haley, D. W., Oberlander, T., Weinberg, J., Solimano, A., Whitfield, M. F., Fitzgerald, C., \& Yu, W. (2005). Neonatal procedural pain exposure predicts lower cortisol and behavioral reactivity in preterm infants in the NICU. Pain, 113, 293-300.

Grunau, R. E., \&Tu, M. T. (2007).Long-term consequences of pain in human neonates. In K. J. S. Anand, B. J. Stevens, \& P. J. McGrath (Eds.), Pain in neonates and infants, 3rd edition (series title: Pain research and clinical management) (pp. 45-55). Philadelphia: Elsevier.

Grunau, R. E., Haley, D. W., Whitfield, M. F., Weinberg, J., Yu, W., \& Thiessen, P. (2007). Altered basal cortisol levels at 3, 6, 8 and 18 months in infants born at extremely low gestational age. Journal of Pediatrics, 150, 151-156. 
*Hermann, C., Hohmeister, J., Demirakça, S., Zohsel, K., \& Flor, H. (2006). Long-term alteration of pain sensitivity in school-aged children with early pain experiences. Pain, 125, 278-285.

*Hohmeister, J., Demirakça, S., Zohsel, K., Flor, H., \& Hermann, C. (2009). Responses to pain in school-aged children with experience in a neonatal intensive care unit: cognitive aspects and maternal influences. European Journal of Pain, 13, 94-101.

*Holsti, L., Grunau, R. E., Oberlander, T. F., \& Whitfield, M.F. (2004). Specific Newborn Individualized Developmental Care and Assessment Program movements are associated with acute pain in preterm infants in the neonatal intensive care unit. Pediatrics, 114, 65-72.

*Holsti, L., Grunau, R. E., Oberlander, T. F., \& Whitfield, M. F. (2005). Prior pain induces heightened motor responses during clustered care in preterm infants in the NICU. Early Human Development, $81,293-302$.

Holsti, L., Grunau, R. E., \& Shany, E. (2011). Assessing pain in preterm infants in the neonatal intensive care unit: moving to a 'brain-oriented' approach. Pain Management, 1, 171-179.

*Holsti, L., Grunau, R. E., Whifield, M. F., Oberlander, T. F., \& Lindh, V. (2006). Behavioral responses to pain are heightened after clustered care in preterm infants born between 30 and 32 weeks gestational age. Clinical Journal of Pain, 22, 757-764.

Johnston, C. C., Fernandes, A. M., \& Campbell-Yeo, M. (2011). Pain in neonates is different. Pain, 152 (3 suppl), S65-S73.

Korner, A. F., Stevenson, D. K., Kraemer, H. C., Spiker, D., Scott, D. T., Constantinou, J., \& Dimiceli, S. (1993). Prediction of the development of low birth weight preterm infants by a new neonatal medical index. Journal of Developmental and Behavioral Pediatrics, 14, 106-111.

Parry, G., Tucker, J., \& Tarnow-Mordi, W. O. (2003). CRIB II: an update of the clinical risk index for babies score. Lancet, 361, 1789-1791.

Ranger, M., Johnston, C. C., \& Anand, K. J. (2007). Current controversies regarding pain assessment in neonates. Seminars in Perinatology, 31, 283-288.

* Rautava, L., Lempinen, A., Ojala, S., Parkkola, R., Rikalainen, H., Lapinleimu, H., Haataja, L., \& Lehtonen, L. (2007). Acoustic quality of cry in very-low-birth-weight infants at the age of 1 1/2 years. Early Human Development, 83, 5-12.
Richardson, D. K., Corcoran, J. D., Escobar, G. J., \& Lee, S. K. (2001). SNAP-II and SNAPPE-II: simplified newborn illness severity and mortality risk scores. Journal of Pediatrics, 138, 92-100.

Richardson, D. K., Gray, J. E., McCormick, M. C., Workman, K., \& Goldmann, D. A. (1993). Score for Neonatal Acute Physiology: a physiologic severity index for neonatal care. Pediatrics, 91, 617-623.

Robertson, C. M., Svenson, L. W., \& Joffres, M. R. (1998).Prevalence of cerebral palsy in Alberta. Canadian Journal of Neurological Science, 25, 117-122.

Slater, R., Fabrizi, L., Worley, A., Meek, J., Boyd, S., \& Fitzgerald, M. (2010). Premature infants display increased noxious-evoked neuronal activity in the brain compared to healthy age-matched term-born infants. NeuroImage, 52, 583-589.

Stevens, B. J., Ridell, R. R. P., Oberlander, T. E., \& Gibbins, S. (2007a). Assessment of pain in neonates and infants. In K. J. S. Anand, B. J. Stevens, \& P. J. McGrath (Eds.), Pain in neonates and infants, 3rd edition (series title: Pain research and clinical management) (pp. 67-90). Philadelphia: Elsevier.

*Stevens, B., McGrath, P., Gibbins, S., Beyene, J., Breau, L., Camfield, C., ... Yamada, J. (2007b). Determining behavioural and physiological responses to pain in infants at risk for neurological impairment. Pain, 127, 94-102.

*Stevens, B., Franck, L., Gibbins, S., McGrath, P.J., Dupuis, A., Yamada, J., ... Ohlsson, A. (2007c). Determining the structure of acute pain responses in vulnerable neonates. Canadian Journal of Nursing Research, 39, 32-47.

*Walker, S. M., Franck, L. S., Fitzgerald, M., Myles, J., Stocks, J., \& Marlow, N. (2009). Long-term impact of neonatal intensive care and surgery on somatosensory perception in children born extremely preterm. Pain,141, 79-87.

*Williams, A. L., Khattak, A. Z., Garza, C. N. \& Lasky, R. E. (2009). The behavioral pain response to heelstick in preterm neonates studied longitudinally: Description, development, determinants, and components. Early Human Development, 85, 369-374.

Zardo, M. S., \& Procianoy, R. S. (2003). Comparison between different mortality risk scores in a neonatal intensive care unit. Revista de Saúde Pública, 37, 591-596. 
